\title{
A bedtime milk snack does not impact RMR, substrate utilisation and appetite the following morning in mildly overweight males
}

\author{
Arthur H. H. Lay ${ }^{1}$, Daniel R. Crabtree ${ }^{2}$, Tom G. Campbell ${ }^{3}$, Gillian M. Dreczkowski ${ }^{1}$, \\ Stuart D. R. Galloway ${ }^{1}$, Kevin D. Tipton ${ }^{1}$ and Oliver C. Witard ${ }^{1 *}$ \\ ${ }^{1}$ Physiology, Exercise and Nutrition Research Group, Faculty of Health Sciences and Sport, University of Stirling, Stirling FK9 \\ $4 L A, U K$ \\ ${ }^{2}$ Centre for Health Science, University of the Highlands and Islands, Inverness IV2 3JH, UK \\ ${ }^{3}$ School of Applied Sciences, Edinburgh Napier University, Edinburgh EH11 4BN, UK \\ (Submitted 29 November 2017 - Final revision received 24 February 2018 - Accepted 24 March 2018)
}

\begin{abstract}
Nighttime eating is often associated with a negative impact on weight management and cardiometabolic health. However, data from recent acute metabolic studies have implicated a benefit of ingesting a bedtime snack for weight management. The present study compared the impact of ingesting a milk snack containing either 10 (BS10) or $30 \mathrm{~g}$ (BS30) protein with a non-energetic placebo (BS0) 30 min before bedtime on next morning metabolism, appetite and energy intake in mildly overweight males (age: $24 \cdot 3$ (SEM 0.8) years; BMI: $27 \cdot 4$ (SEM 1.1) kg/m²). Next morning measurements of RMR, appetite and energy intake were measured using indirect calorimetry, visual analogue scales and an ad libitum breakfast, respectively. Bedtime milk ingestion did not alter next morning RMR (BS0: 7822 (SEM 276) kJ/d, BS10: 7482 (SEM 262) kJ/d, BS30: 7851 (SEM 261) kJ/d, $P=0 \cdot 19)$ or substrate utilisation as measured by RER $(P=0 \cdot 64)$. Bedtime milk ingestion reduced hunger $(P=0 \cdot 01)$ and increased fullness $(P=0 \cdot 04)$ during the evening immediately after snack ingestion, but elicited no effect the next morning. Next morning breakfast (BS0: 2187 (sEM 365 ) kJ, BS10: 2070 (sEm 336) kJ, BS30: 2582 (SEM 384) kJ, $P=0.21$ ) and 24 h post-trial $(P=0.95)$ energy intake was similar between conditions. To conclude, in mildly overweight adults, compared with a non-energetic placebo, a bedtime milk snack containing 10 or $30 \mathrm{~g}$ of protein does not confer changes in next morning whole-body metabolism and appetite that may favour weight management.
\end{abstract}

Key words: Milk: Bedtime snacks: RMR: Appetite

Several observational studies reveal that eating late in the day, for example immediately before bedtime, is associated with increased overall daily energy intake ${ }^{(1)}$, poor weight management ${ }^{(2)}$, and increased CVD risk ${ }^{(3)}$. Consistent with this notion, physiological data exist to demonstrate that energy intake in the hours immediately leading up to bedtime results in a lower acute diet-induced thermogenesis ${ }^{(4)}$ and a reduced feeling of satiation compared with energy intake in the morning or afternoon ${ }^{(1)}$. Thus, it is intuitive that over a chronic time period, a dietary pattern in which energy intake is prioritised close to bedtime may promote a positive energy balance and weight gain.

Conversely, there are emerging data from acute metabolic studies indicating that consumption of lower energy and single macronutrient snacks $30 \mathrm{~min}$ before bedtime may confer favourable outcomes with regards to whole-body metabolism and appetite ${ }^{(5)}$. Collectively, these bedtime snack studies have focused primarily on comparing the impact of acute ingestion of the individual macronutrient constituents of milk (whey protein, casein protein and carbohydrate) on next morning RMR, substrate utilisation and appetite ${ }^{(6-10)}$. For instance, the consumption of $30 \mathrm{~g}$ of whey protein, $30 \mathrm{~g}$ of casein protein or $33 \mathrm{~g}$ of carbohydrate (equivalent to an energy intake of $586-627 \mathrm{~kJ}$ ) $30 \mathrm{~min}$ before bedtime was reported to increase next morning $\mathrm{RMR}$ in active young men ${ }^{(7)}$. In addition, next morning fat oxidation rates were increased with bedtime casein ingestion compared with whey and carbohydrate ingestion ${ }^{(7)}$. The authors speculated that the lower insulin response elicited by casein ingestion $^{(11)}$ may explain the higher rates of fat oxidation the next morning in the bedtime casein snack condition ${ }^{(7)}$. Furthermore, a subsequent study in overweight and obese females reported an increased next morning satiety and decreased desire to eat with bedtime whey, casein, or carbohydrate ingestion compared with the omission of a bedtime snack $^{(6)}$. Interestingly, this study reported elevated fasting blood insulin concentrations the following morning in the bedtime snack conditions, despite no changes in fasting glucose concentrations. Whereas these data in overweight and obese females imply that an acute bedtime snack, irrespective of

Abbreviations: $\mathrm{BS} 0,0 \mathrm{~g}$ protein; BS10, $10 \mathrm{~g}$ protein; BS30, $30 \mathrm{~g}$ protein.

* Corresponding author: O. C. Witard, email oliver.witard@stir.ac.uk 
content, may increase next morning cardiometabolic risk by increasing insulin resistance ${ }^{(6)}$, the same increase in fasting blood insulin concentrations was not observed in obese males ${ }^{(8)}$. Hence, a growing body of scientific evidence from acute metabolic studies supports the notion that a low energy snack (approximately $586-627 \mathrm{~kJ}$ ) before bedtime may be beneficial for weight management, but with unclear impact on the acute cardiometabolic risk the following morning in overweight and obese individuals.

Casein protein is commonly perceived to be an ideal bedtime snack given its slower digestion properties that allows for a sustained elevation in plasma amino acid concentrations for the duration of sleep ${ }^{(5,12)}$. Nonetheless, based on findings from acute studies, whey protein and carbohydrate also appear to be important components of a bedtime snack as an increase in next morning RMR has been shown to be comparable to casein protein in active young men ${ }^{(7)}$. Given that milk is a proteindense foodstuff, consisting of $80 \%$ casein and $20 \%$ whey protein, and contains carbohydrate ${ }^{(13)}$, in theory milk, may be considered an ideal bedtime snack for increasing next morning RMR because of its macronutrient composition. Readily available in both fluid and powder form, milk provides a more practical and economically viable bedtime snack compared with an isolated (or hydrolysed) whey or casein protein supplement ${ }^{(14)}$. Moreover, within an acute study setting, the provision of milk as a mid-day snack, or as part of a standardised breakfast, has been shown to be effective in decreasing perceived appetite when compared with ingestion of water and beverages comprised primarily of carbohydrate ${ }^{(15,16)}$. However, to our knowledge, only a single study to date in female athletes has examined the impact of bedtime milk ingestion, administered in chocolate milk form, compared with a non-energetic placebo and observed an increase in RMR and reduction in appetite the following morning ${ }^{(10)}$. A logical follow-up study is to investigate the impact of bedtime consumption of a mixed macronutrient food source such as milk on next morning RMR, substrate utilisation, and appetite in healthy, overweight adults.

Evidence regarding the optimal bedtime protein dose required to effectively modulate RMR and appetite the following morning also remains unknown. Interestingly, the intake of $\sim 30 \mathrm{~g}$ of protein during the day has been shown to induce greater diet-induced thermogenesis, modulate appetite, and enhance satiety $^{(17,18)}$. However, to date, no acute study has examined whether a protein dose less than $30 \mathrm{~g}$ confers a similar increase in RMR and modulatory effect on appetite the following morning.

Accordingly, the primary aim of this acute metabolic study was to compare the impact of bedtime skimmed milk ingestion to a non-energetic placebo on next morning RMR, substrate utilisation, subjective appetite ratings, subsequent energy intake, and insulin and glucose responses in healthy, mildly overweight young men. The secondary aim was to determine the dose-response relationship between bedtime milk ingestion and next morning RMR, substrate utilisation, appetite and energy intake. We hypothesised that ingestion of the bedtime milk beverage containing $30 \mathrm{~g}$ of protein would increase next morning RMR, reduce appetite, and increase fat oxidation rates to a greater extent than a milk beverage containing $10 \mathrm{~g}$ of protein or a non-energetic control. We also hypothesised that next morning fasting insulin and glucose responses would be similar between bedtime snack conditions in this cohort of healthy, mildly overweight males.

\section{Methods}

\section{Participants and ethics approval}

In all, twelve healthy, mildly overweight but weight stable, and active but untrained males participated in the present study. A priori, we conducted a power calculation (GPower version 3 software) of appropriate sample size based on previous published data ${ }^{(10)}$ that measured, on average, a $5 \%$ higher RMR the following morning after bedtime ingestion of chocolate milk $v$. placebo using the same indirect calorimetry technique conducted in the present study. By setting statistical power (1- $\beta$ err prob) at $0.8, \alpha$ error probability at 0.05 and effect size (Cohen's $d$ ) at 1.4 (based on previous data ${ }^{(10)}$ ), our power calculation revealed a minimum sample size of ten participants (using a cross-over research design) would be necessary to detect a statistical difference in RMR between milk and placebo treatment conditions. Exclusion criteria included any known diagnosis of CVD, stroke, diabetes mellitus and thyroid or kidney dysfunction. Participants taking medications that may affect appetite, taste and smell were excluded. Smokers and those with lactose intolerance or a dislike of dairy products also were excluded. Baseline anthropometric parameters including age, height, weight, BMI, waist and hip circumferences, and the sum of five skinfolds (triceps, biceps, subscapular, iliac crest, calf) were measured before the start of experimental trials (Table 1). The present study was conducted according to guidelines laid down in the Declaration of Helsinki and all procedures involving human subjects were approved by the University of Stirling, Faculty of Health Sciences and Sport Research Ethics Committee. Written informed consent and health questionnaires were obtained from all participants before participation.

\section{Protocol overview}

Each experimental trial was conducted over $2 \mathrm{~d}$ (see Fig. 1 for protocol overview). Consistent with similar previous studies $^{(6,7,9)}$, a washout period of $\geq 4 \mathrm{~d}$ was standardised between trials. On day 1, participants consumed a standardised evening meal at 19.30 hours and then a bedtime snack at 22.30 hours on the night before the morning laboratory visit. Subjective appetite and thirst were assessed before and after the

Table 1. Participant characteristics

(Mean values with their standard errors; $n$ 12)

\begin{tabular}{lrr}
\hline & Mean & SEM \\
\hline Age $($ years $)$ & 24.3 & $0 \cdot 8$ \\
Height $(\mathrm{cm})$ & 182.0 & $2 \cdot 0$ \\
Weight $(\mathrm{kg})$ & 91.0 & 4.4 \\
BMl $\left(\mathrm{kg} / \mathrm{m}^{2}\right)$ & 27.4 & $1 \cdot 1$ \\
Waist circumference $(\mathrm{cm})$ & $90 \cdot 7$ & $3 \cdot 2$ \\
Hip circumference $(\mathrm{cm})$ & 106.9 & $2 \cdot 7$ \\
Skinfolds $(\mathrm{mm})^{*}$ & 92.6 & $13 \cdot 2$ \\
\hline
\end{tabular}

* Sum of skinfolds included triceps, biceps, subscapular, iliac crest and calf. 
(a)
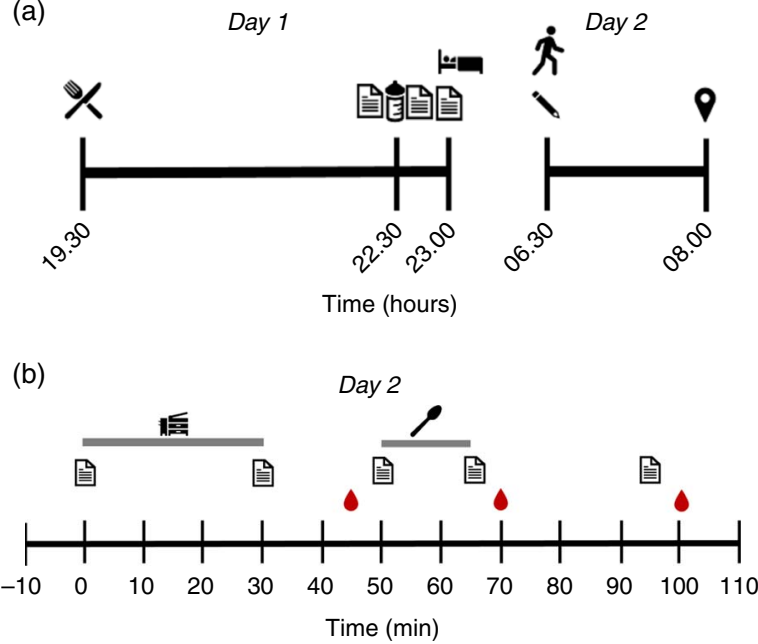

Fig. 1. Schematic diagram of study protocol on (a) day 1 and day 2 before arriving at the laboratory and (b) during the trial on day 2. A standardised dinner was consumed at 19.30 hours, followed by the bedtime snack at 22.30 hours. Participants went to sleep at 23.00 hours and woke up at 06.30 hours the next day. Participants arrived at the laboratory at 08.00 hours and rested in the supine position for $10 \mathrm{~min}$. Metabolic measurements were completed via indirect calorimetry for $20 \mathrm{~min}$, which was proceeded by the 15-min ad libitum breakfast. The appetite and thirst questionnaire was completed before and after both the metabolic measurements and breakfast. The first and second blood samples were conducted before breakfast and after breakfast. The final questionnaire and blood sample were taken $30 \mathrm{~min}$ after breakfast. $\%$ Standardised dinner; 管, appetite and thirst questionnaire; 会, bedtime snack; \, Leeds Sleep Evaluation Questionnaire; $\boldsymbol{\vartheta}$, arrival at laboratory; 佔, indirect calorimetry; , blood sample; $\boldsymbol{\rho}$, ad libitum breakfast of cornflakes and semiskimmed milk.

bedtime snack and before the standardised bedtime at 23.00 hours. Overnight, participants wore actigraphy devices on their wrists for the assessment of sleep quality.

The next morning, participants woke up at 06.30 hours and immediately completed a questionnaire to assess sleep quality before attending the laboratory. Sleep quality (including sleep duration) was also assessed objectively using actigraphy (see the 'Measurements of sleep quality' section). Participants arrived at the laboratory fasted at 08.00 hours, having abstained from moderate-to-high intensity exercise, alcohol intake, and caffeine consumption for $24 \mathrm{~h}$, and rested supine on a bed for $10 \mathrm{~min}$. Subjective appetite and thirst were assessed at the end of the $10 \mathrm{~min}$ rest period. Metabolic measurements were then completed using indirect calorimetry for $30 \mathrm{~min}$. Subsequently, subjective appetite and thirst were assessed again followed by collection of the first blood sample (08.45-08.50 hours) and the ad libitum breakfast. Subjective appetite and thirst also were assessed before and after breakfast and again $30 \mathrm{~min}$ after breakfast. Additional blood samples were collected immediately after the $15 \mathrm{~min}$ breakfast period (09.05-09.10 hours) and 30 min (09.35-09.40 hours) after the end of breakfast.

\section{Bedtime beverage treatments}

The study was randomised and cross-over in design. Treatments were double-blind except for the non-energetic placebo (BS0), which was water. A third party, not involved in other aspects of the study, prepared the beverages in advance and randomised the
Table 2. Energy and macronutrient content of bedtime snack treatments

\begin{tabular}{lccr}
\hline & BS0 & BS10 & BS30 \\
\hline Skimmed milk powder $(\mathrm{g})$ & 0 & 28 & 84 \\
Energy (kJ) & 0 & 410 & 1234 \\
Protein (g) & 0 & 10 & 30 \\
Casein (g) & 0 & 8 & 24 \\
Whey (g) & 0 & 2 & 6 \\
Carbohydrate (g) & 0 & 14 & 42 \\
Fat $(\mathrm{g})$ & 0 & 0.2 & 0.5 \\
\hline
\end{tabular}

BS0, placebo; BS10, $10 \mathrm{~g}$ protein; BS30, $30 \mathrm{~g}$ protein.

treatments in a counterbalanced order, with at least $4 \mathrm{~d}$ separating trials. Treatments were given to participants as pre-weighed Tesco $^{\odot}$ instant dried skimmed milk powder (Tesco Stores Ltd) in opaque plastic beverage bottles instead of fluid milk to ensure treatments were isovolumetric. Participants were instructed to add $400 \mathrm{ml}$ of water to dissolve the skimmed milk powder thoroughly by shaking the bottle before ingestion at home. Macronutrient breakdown and energy content of treatments are described in Table 2. The treatment condition containing $10 \mathrm{~g}$ of protein (BS10) was chosen to mimic the approximate amount of protein in a typical glass of milk. The treatment with the highest amount of protein (BS30) was chosen to meet the $30 \mathrm{~g}$ protein threshold postulated to be required to suppress appetite ${ }^{(18)}$ and to match the protein dose administered in previous bedtime snack studies ${ }^{(6-9)}$. Participants were given an empty bottle for BSO and filled it with $400 \mathrm{ml}$ of tap water to be consumed at the time of the bedtime beverage.

\section{Diet control}

Participants completed a weighed food diary for three separate evening meals before beginning the study. Energy and macronutrient intakes were calculated using dietary analysis software (Nutritics Academic Edition version 4.267; Nutritics). The average energy intake of the three evening meals was used to determine the total energy content of the standardised evening meal. The standardised evening meal was designed to provide the same macronutrient breakdown of diets in UK adults according to the National Diet and Nutrition Survey 2008/20092011/2012 (carbohydrate: $50 \%$; fat: $32 \%$, protein $18 \%)^{(19)}$. The standardised evening meal consisted of Tesco $^{\odot}$ Fusilli Pasta Twists, Tesco ${ }^{\odot}$ Bolognese Pasta Sauce, Tesco ${ }^{\odot}$ Beef Lean Steak Mince 5\% Fat (Tesco Stores Ltd) and olive oil. The ingredients were supplied to the participants and instructions were provided to prepare the meal at home. Compliance was verified verbally and by the return of empty food containers.

Participants also kept a 2 - $\mathrm{d}$ food and activity diary $48 \mathrm{~h}$ before the first experimental trial and were asked to replicate the same food intake and activity in the $48 \mathrm{~h}$ before the subsequent trials. No other food or drink was permitted after consumption of the bedtime beverage the night before the morning trials. Participants were asked to consume $300 \mathrm{ml}$ of water in the morning before visiting the laboratory.

\section{Metabolic measurements}

Oxygen consumption and carbon dioxide production were measured via indirect calorimetry (Oxycon Pro; Cardinal 
Health) using a ventilated metabolic hood placed over the participant's head. Before starting the measurements, a calibration program within the software application accompanying the metabolic cart (LabManager, version 5.30.0) was used to determine ambient conditions (temperature, relative humidity and barometer pressure). Volume calibration was completed manually using a 3 litre calibration pump and gas analyzer calibration was completed using verified gases of known concentrations $\left(16 \% \mathrm{O}_{2}\right.$ and $\left.5 \% \mathrm{CO}_{2}\right)$. Measurements were completed with participants resting supine on a bed in a quiet and temperaturecontrolled room $\left(20-24^{\circ} \mathrm{C}\right)$. Gas exchange was measured continuously for $30 \mathrm{~min}$ and data were captured every $30 \mathrm{~s}$. The software application determined the RER and calculated the RMR using the formula derived by Weir ${ }^{(20)}$. Only the final $20 \mathrm{~min}$ of the data collection period was used for analysis to ensure participants were at a physiological steady state.

\section{Subjective assessment of hunger, fullness, desire to eat and thirst}

Hunger, fullness, desire to eat, and thirst were assessed subjectively using a validated visual analogue scale (VAS) ${ }^{(21)}$. The questions accompanying the VAS were 'How hungry do you feel?', 'How full do you feel?', 'How strong is your desire to eat now?' and 'How thirsty are you?'. The horizontal lines were anchored by the statements 'Not at all hungry/full/thirsty' and 'As hungry/full/thirsty as I have ever felt' at each end. For the desire to eat, the statements 'Not at all' and 'Extremely' were used at each end of the horizontal line. Participants placed a vertical mark on a $100-\mathrm{mm}$ horizontal scale to rate how they felt regarding each sensation. Participants were instructed not to refer to previous scales when completing each new set of scales.

\section{Ad libitum breakfast and $24 \mathrm{~h}$ post-trial energy intake}

Participants were given $15 \mathrm{~min}$ to consume an ad libitum breakfast at a dining table in an isolated area of the research kitchen to minimise external distractions. Participants were provided a packet of Kellogg's Corn Flakes ${ }^{\circledR}$ (Kellogg Company of Great Britain Ltd), a 500-ml jar of semi-skimmed milk, and instructed to eat as little or as much of each breakfast item as they wanted until they were satisfied. If participants finished eating before the allotted $15 \mathrm{~min}$, they remained seated at the table. The packet of Kellogg's Corn Flakes ${ }^{\circledR}$ (1582 kJ/100 g) was weighed before and after the ad libitum breakfast to determine the amount the participant consumed. The volume of semiskimmed milk (Tesco ${ }^{\circledR}$ British semi skimmed milk, $209 \mathrm{~kJ}$ $(50 \mathrm{kcal}) / 100 \mathrm{ml}$; Tesco Stores Ltd) remaining in the jar was measured in a graduated cylinder to determine volume consumed. All participants answered 'yes' to whether they would like cornflakes and milk for breakfast in the pre-study questionnaire. Participants were not informed that the energy intake of the cereal was being measured, as this information may have influenced their eating habits.

At the end of each trial, participants were instructed to keep a detailed food record of all food and beverages consumed in the 24-h post-trial period. The food records were analysed using dietary analysis software. Ten participants were included in the analysis of energy intake in the $24 \mathrm{~h}$ post-trial period as two participants were unable to provide complete food records.

\section{Measurements of sleep quality}

Given that sleep restriction has been associated with reduced next morning $\mathrm{RMR}^{(22)}$, objective and subjective measurements of sleep were assessed to investigate the acute effect of bedtime milk ingestion on sleep. The MotionWatch $8^{\odot}$ (CamNTech Ltd) tri-axial wrist-worn actigraphy device was used to obtain three objective measurements of sleep quality - actual sleep time, sleep latency, and fragmentation index. Actual sleep time was defined as total minutes categorised as sleep by the actigraphy device and the accompanying software (MotionWare, 1.125; CamNTech Ltd.). Sleep latency was defined as the time between 'lights out' and 'fell asleep' time points. Fragmentation index, expressed as the sum of total mobile time and immobile bouts not exceeding $1 \mathrm{~min}$ in duration, is a measure of disruption to sleep periods used as a marker of sleep quality, with a higher value indicating lower quality sleep.

Participants completed the Leeds Sleep Evaluation Questionnaire (LSEQ) immediately upon waking on the morning of the experimental trials for subjective measurements of sleep quality. The LSEQ was validated in individuals aged 18-49 years and consists of ten VAS questions that evaluate four domains of sleep: the ease of getting to sleep, the perceived quality of sleep, the ease of awakening from sleep, and behaviour following wakefulness ${ }^{(23)}$. Participants were asked to place a mark on the $100-\mathrm{mm}$ line based on how they felt between two extremes, for example 'less sleepy than usual' and 'more sleepy than usual'. The scores were averaged to give a score for each domain.

\section{Blood sampling and analyses}

A cannula (Becton, Dickinson \& Company) was inserted into a forearm vein for blood sampling. At each timepoint, $10 \mathrm{ml}$ of blood was dispensed evenly between lithium heparin or clot activator vacutainer tubes. Within $120 \mathrm{~min}$, lithium heparin vacutainers were centrifuged at $3500 \mathrm{rpm}$ at $4{ }^{\circ} \mathrm{C}$ and plasma aliquots were dispensed into Eppendorf tubes. Clot activator vacutainers were allowed to clot for $60 \mathrm{~min}$ at room temperature before centrifugation and dispensing serum aliquots into Eppendorf tubes. Plasma and serum samples were stored at $-80^{\circ} \mathrm{C}$ for future analysis of glucose and insulin concentrations, respectively. Plasma glucose concentrations were analysed with use of an automated analyser (ILab Aries; Instrumentation Laboratory) and serum insulin concentrations were analysed with use of a commercially available ELISA kit (Demeditec Diagnostics $\mathrm{GmbH}$ ) according to manufacturer's instructions. The HOMA2 Calculator version 2.2.3 ${ }^{(24)}$ was used to determine the homoeostatic model assessment of insulin resistance (HOMA-IR) value. The averages of duplicate samples were used for data analysis. The intra-assay CV and inter-assay CV for insulin concentrations were 8.5 and $10 \cdot 8 \%$, respectively. Two participants were unable to provide blood samples for all three trials; therefore ten participants were included in the final analysis of blood samples. 


\section{Data presentation and statistical analysis}

Statistical analyses were conducted using IBM $^{\circledR}$ SPSS $^{\circledR}$ Statistics software package version 23 (IBM Corporation). AUC was calculated using the trapezoidal method with the baseline set as the value measured immediately after bedtime snack ingestion for the evening period and at $0 \mathrm{~min}$ for the next morning period (see Fig. 1). One-way repeated-measures ANOVA were conducted to examine differences in RMR, RER, estimated carbohydrate oxidation and fat oxidation rates, energy intake at ad libitum breakfast, $24 \mathrm{~h}$ post-trial energy intake, HOMA-IR, the AUC of subjective appetite and thirst assessments, actual sleep time, sleep latency, fragmentation index and the four domains of sleep in the LSEQ. Two-way repeated-measures ANOVA was conducted to test for treatment, time and treatment $\times$ time interaction effects on subjective assessments of hunger, fullness, desire to eat and thirst and also glucose and insulin concentrations. Where a significant treatment and/or interaction effect was detected, Bonferroni post hoc test was used to determine specific differences for both one-way and two-way repeated-measures ANOVA. Statistical significance was determined at an $\alpha$ level of $P<0.05$, and data were reported as mean with standard errors unless specified otherwise.

\section{Results}

\section{Pre-trial dietary intake}

Analysis of the pre-trial 2-d food diary revealed a daily mean energy intake of $26.3(\operatorname{sem} 3.4) \mathrm{kJ} / \mathrm{kg} / \mathrm{d}$ and a macronutrient breakdown of 45.5 (SEM 2.5 ) \% carbohydrate, 19.2 (SEM 1.2 ) \% protein and $35 \cdot 3$ (SEM 1.7 ) \% fat.

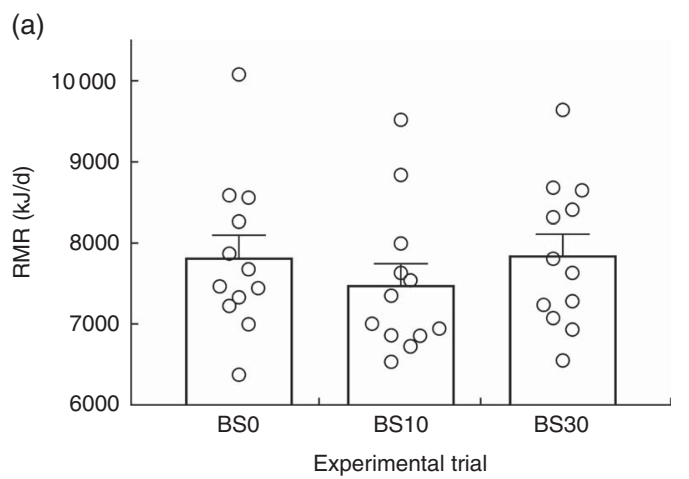

\section{Metabolic measurements}

There was no significant effect of bedtime snack treatment on next morning RMR $(P=0.19)$ (Fig. 2(a)) or RER $(P=0 \cdot 64)$ (Fig. 2(b)). Likewise, there was no significant effect of bedtime snack treatment on estimated carbohydrate $(P=0.51)$ or fat $(P=0 \cdot 17)$ oxidation rates (Fig. 2(c)).

\section{Subjective assessment of hunger, fullness, desire to eat, and thirst}

Subjective assessments of hunger, fullness, and desire to eat are represented in Fig. 3. A significant main effect of bedtime snack treatment was observed on subjective measurements of hunger $(P=0.01)$ and fullness $(P=0.04)$ during the evening period after bedtime milk ingestion. Hunger ratings for BS30 were significantly lower than BS0 during the evening at $5(P=0.04)$ and $30 \mathrm{~min}(P=0 \cdot 001)$ after bedtime milk ingestion, but were only significantly lower at $30 \mathrm{~min}$ for BS10 $v$. BSO $(P=0 \cdot 01)$ (Fig. 3(a)). Evening fullness ratings for BS30 were significantly higher than BS0 at $30 \mathrm{~min}(P=0.007)$ after bedtime milk ingestion, whereas BS10 fullness ratings were higher than BS0 at $5 \mathrm{~min}(P=0.02)$ (Fig. 3(b)). There were no differences between BS30 and BS10 in subjective hunger or fullness during the evening after bedtime milk ingestion $(P>0.05)$.

There was a trend for a significant effect of bedtime snack on the next morning rating of fullness $(P=0.07)$, but not next morning hunger $(P=0 \cdot 60)$. No significant effect of bedtime snack was observed on the desire to eat or thirst both during the evening after ingestion (desire to eat: $P=0.21$; thirst: $P=0.71$ )

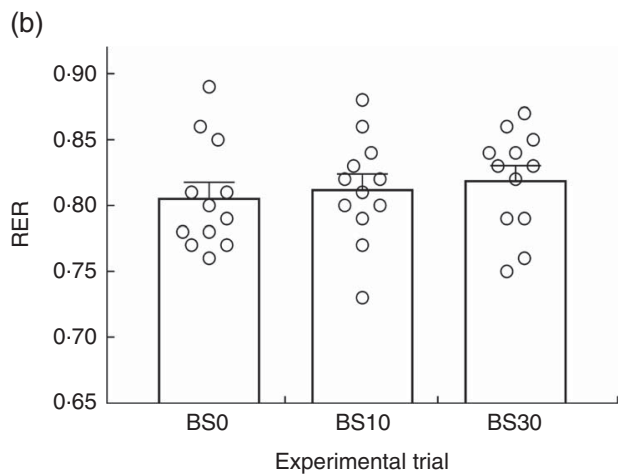

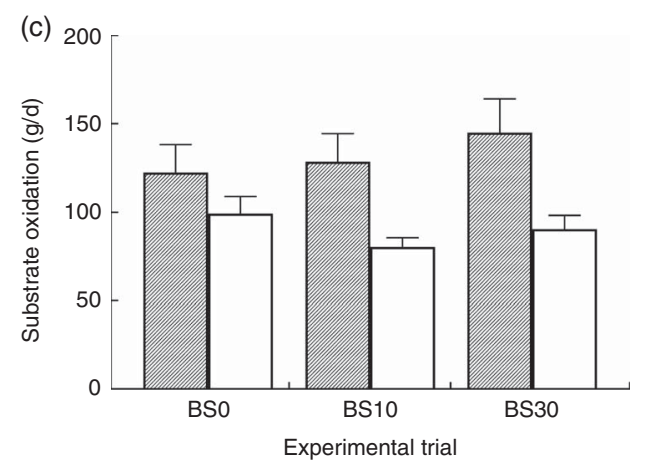

Fig. 2. Next morning (a) RMR, (b) respiratory exchange ratio (RER) and (c) carbohydrate (שי $)$ and fat ( $\square$ ) oxidation following bedtime milk ingestion. Values are means with their standard errors. No significant main effect of bedtime snack was observed for all measurements $(P>0.05$, one-way repeated-measures ANOVA). BSO, $0 \mathrm{~g}$ protein; BS10, $10 \mathrm{~g}$ protein; BS30, $30 \mathrm{~g}$ protein. 
(a)

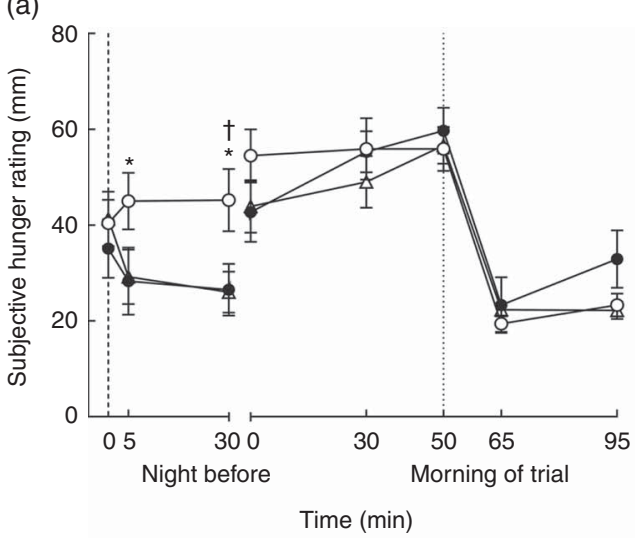

(b)

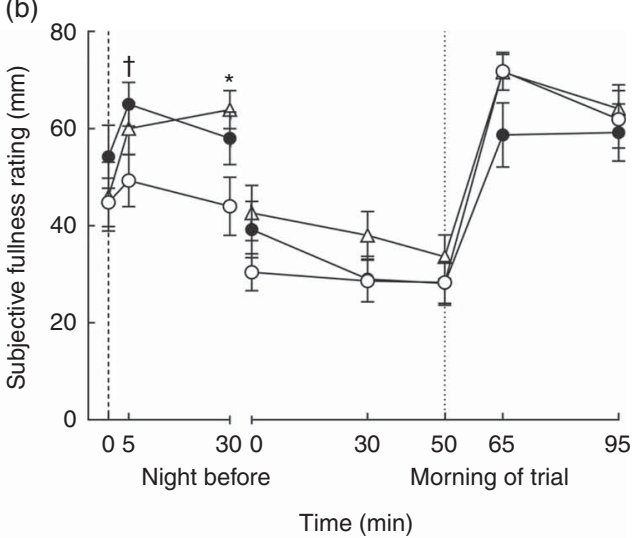

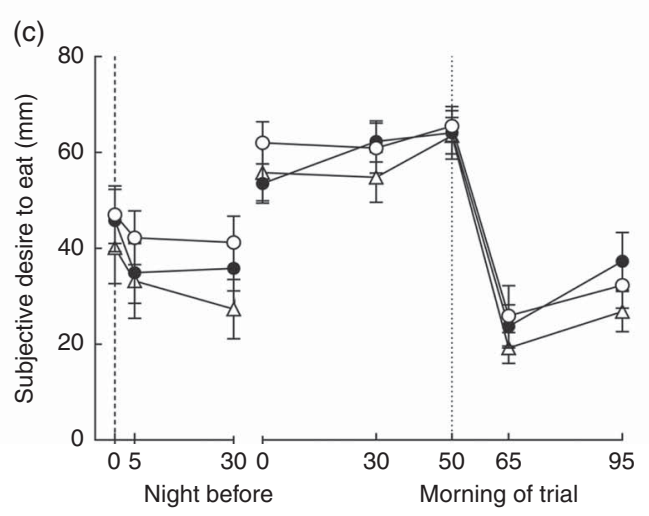

Time (min)

Fig. 3. Next morning subjective (a) hunger, (b) fullness and (c) desire to eat following bedtime milk ingestion. -----, the time point when bedtime milk was ingested. Values are means with their standard errors. ........., time when ad libitum breakfast was ingested; $-\mathrm{O}-, 0 \mathrm{~g} \mathrm{protein}(\mathrm{BS} 0) ;-\bigcirc, 10 \mathrm{~g}$ protein $(\mathrm{BS} 10) ;-\triangle-30 \mathrm{~g}$ protein (BS30). Data were analysed using a two-way (bedtime snack $\times$ time) repeated-measures ANOVA. Measurements from the night before and morning of trial were analysed separately. At night, there was a significant main effect of bedtime snack on hunger and fullness $(P<0.05)$. The following morning, there was a trend towards a significant effect of bedtime snack on fullness $(P=0.07)$, but no significant effect was observed for hunger and desire to eat $(P>0.05)$. Bonferroni's post hoc test was conducted to determine differences between means. * Mean value was significantly different between BS0 and BS30. $†$ Mean value was significantly different between BSO and BS10.

or the following morning (desire to eat: $P=0.42$; thirst: $P=0 \cdot 91$ )

Subjective appetite and thirst measurements also were expressed as AUC calculated over periods between bedtime snack ingestion and sleep, and from 0 to 95 min on the morning of the trials (Fig. 4). There was a significant effect of bedtime snack treatment on the AUC for hunger $(P=0.006)$ and fullness $(P=0.02)$ during the evening period. The bedtime snack treatment had no effect on AUC for hunger measured the following morning $(P=0.62)$, but there was a trend for a significant effect on the AUC of fullness the following morning $(P=0.07)$. No effect of bedtime snack treatment was observed for AUC of desire to eat and thirst calculated over the evening period (desire to eat: $P=0.21$; thirst: $P=0 \cdot 23$ ) or the following morning (desire to eat: $P=0 \cdot 39$; thirst: $P=0 \cdot 91$ ) (data not shown for thirst).

\section{Ad libitum breakfast and $24 \mathrm{~h}$ post-trial energy intake}

There was no significant effect of bedtime snack treatment on energy intake at the ad libitum breakfast (BSO: 2187 (SEM 356) kJ, BS10: 2070 (sem 336) kJ, BS30: 2582 (sEm 384) kJ, $P=0 \cdot 21$ ). Likewise, bedtime snack did not have a significant effect on $24 \mathrm{~h}$ post-trial energy intake when expressed per $\mathrm{kg}$ body weight (BSO: 105 (SEM 16) kJ/kg, BS10: 108 (SEM 11) kJ/kg, BS30: 108 (sEM 16) kJ/kg, $P=0.95$ ).

\section{Blood glucose and insulin concentrations}

There was no significant bedtime snack and time interaction on next morning plasma glucose $(P=0.60)$ or serum insulin $(P=0.57)$ concentrations. Bedtime snack did not have a significant effect on next morning plasma glucose $(P=0 \cdot 61)$, serum insulin $(P=0.56)$ or HOMA-IR $(P=0.85)$ (Table 3$)$. A main effect of time on plasma glucose and serum insulin concentrations $(P<0 \cdot 01)$ was observed.

\section{Sleep measurements}

As measured by the actigraphy devices, there was no significant effect of bedtime snack treatment on actual sleep time (BS0: 351 (SEM 9) min, BS10: 366 (SEM 12) min, BS30: 333 (SEM 20) min, $P=0 \cdot 18$ ). Likewise, no significant effect of bedtime snack treatment on sleep latency was observed (BSO: $20 \cdot 3$ (SEM 7.0) min, BS10: 23.7 (SEM 8.8) min, BS30: $30 \cdot 3$ (SEM 11.6) $\mathrm{min}, P=0.76$ ). There also was no significant effect of bedtime snack treatment on fragmentation index (BS0: $28 \cdot 8$ (SEM 2.4), BS10: $29 \cdot 2$ (SEM 4.9) 
(A)

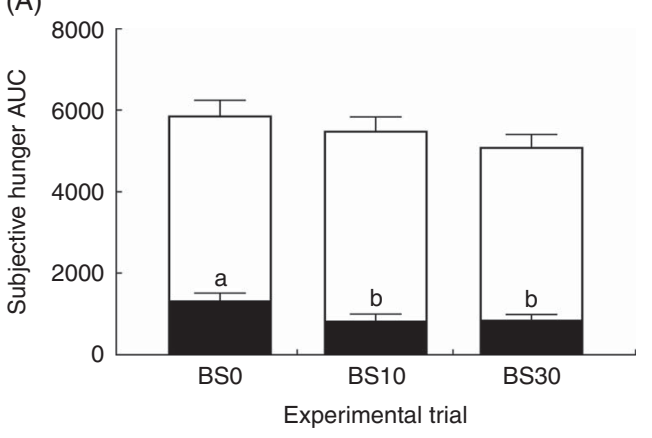

(B)

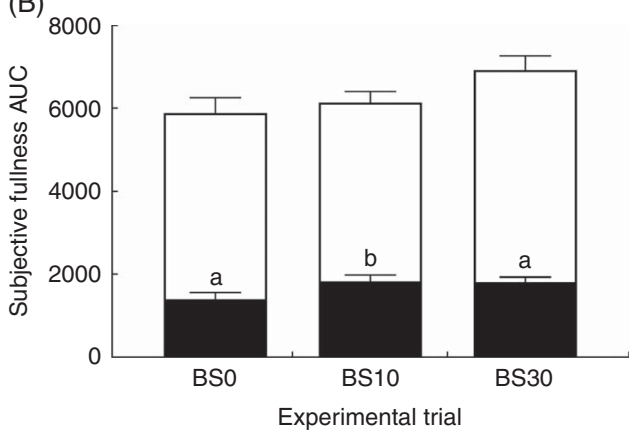

Table 3. Plasma glucose and serum insulin concentrations

(Mean values with their standard errors; $n 10$ )

\begin{tabular}{|c|c|c|c|c|c|c|}
\hline & \multicolumn{2}{|c|}{$\begin{array}{l}\text { Before ad libitum } \\
\text { breakfast }\end{array}$} & \multicolumn{2}{|c|}{$\begin{array}{l}\text { After ad libitum } \\
\text { breakfast }\end{array}$} & \multicolumn{2}{|c|}{$\begin{array}{c}30 \text { min after ad libitum } \\
\text { breakfast }\end{array}$} \\
\hline & Mean & SEM & Mean & SEM & Mean & SEM \\
\hline \multicolumn{7}{|c|}{ Plasma glucose (mmol/l) } \\
\hline BSO & $4.7^{\mathrm{a}}$ & 0.3 & $6 \cdot 0^{\mathrm{a}, \mathrm{b}}$ & 0.7 & $6.9^{b}$ & 0.5 \\
\hline BS10 & $4.4^{\mathrm{a}}$ & 0.2 & $5 \cdot 6^{\mathrm{a}}$ & 0.6 & $6 \cdot 6^{\mathrm{a}}$ & 0.8 \\
\hline BS30 & $4 \cdot 5^{\mathrm{a}}$ & 0.3 & $5 \cdot 5^{\mathrm{a}, \mathrm{b}}$ & 0.5 & $7 \cdot 0^{\mathrm{c}}$ & 0.7 \\
\hline \multicolumn{7}{|c|}{ Serum insulin $(\mathrm{pmol} / \mathrm{l})$} \\
\hline BSO & $63 \cdot 6^{\mathrm{a}}$ & 5.8 & $311.5^{\mathrm{a}, \mathrm{b}}$ & 90.7 & $504 \cdot 6^{\mathrm{b}}$ & $68 \cdot 0$ \\
\hline BS10 & $69.5^{\mathrm{a}}$ & $8 \cdot 3$ & $254.5^{\mathrm{b}}$ & 64.4 & $423.2^{b}$ & $50 \cdot 7$ \\
\hline BS30 & $63.8^{\mathrm{a}}$ & 4.7 & $244 \cdot 7^{b}$ & $56 \cdot 2$ & $506 \cdot 9^{c}$ & 79.4 \\
\hline
\end{tabular}

$\mathrm{BS} 0$, placebo; BS10, $10 \mathrm{~g}$ protein; $\mathrm{BS} 30,30 \mathrm{~g}$ protein.

a,b,c Mean values across a row with unlike superscript letters were significantly different from each other $(P<0.05$, repeated-measures two-way ANOVA, Bonferroni post hoc test).

and BS30: 35.9 (SEM 5.5), $P=0.41$ ). Similarly, bedtime snack treatment had no significant effect on any of the four domains of subjective sleep in the LSEQ (data not shown): 'getting to sleep' $(P=0.95)$, 'quality of sleep' $(P=0.66)$, 'awake following sleep' $(P=0.77)$ and 'behaviour following awakening' $(P=0 \cdot 86)$.

\section{Discussion}

The primary aim of the present study was to investigate the influence of bedtime skimmed milk ingestion on acute changes in whole-body metabolism and appetite the following morning in mildly overweight males. The main finding was that bedtime ingestion of a milk snack containing either 10 or $30 \mathrm{~g}$ of protein did not increase next morning RMR compared with a nonenergetic placebo. In addition, next morning RER, as well as carbohydrate oxidation and fat oxidation rates, were similar between milk and non-energetic placebo conditions. Whereas the bedtime milk conditions tended $(P=0.07)$ to increase subjective fullness the next morning, no differences in hunger and desire to eat were observed between milk and nonenergetic placebo conditions. Accordingly, energy intake at an ad libitum breakfast the next morning and $24 \mathrm{~h}$ post-trial was similar between conditions. Hence, refuting our original hypothesis, bedtime milk ingestion failed to increase RMR and fat oxidation or reduce appetite the next morning compared with a non-energetic placebo in mildly overweight males. Consistent with our second hypothesis, next morning fasting insulin and glucose responses were similar between bedtime snack conditions in this cohort of healthy, mildly overweight males.

In the present study, we anticipated a dose-dependent increase in next morning RMR with bedtime milk intake due, at least in part, to differences in the protein and energy content of test drinks. The two primary factors known to influence dietinduced thermogenesis are protein and energy content, with protein estimated to contribute up to $30 \%$ of diet-induced thermogenesis ${ }^{(25)}$. Hence, previous bedtime snack studies have proposed an energy-induced increase in thermogenesis to be a key mechanism behind the increase in next morning RMR following bedtime snack ingestion ${ }^{(6,7,10)}$. In the present study, 
the BS10 condition was chosen to mimic the $7-10 \mathrm{~g}$ of protein contained in a typical glass of milk and was similar to the $12 \mathrm{~g}$ of protein in the bedtime chocolate milk intervention administered previously by Ormsbee et al. ${ }^{(10)}$. In addition to being higher in protein and energy content than BS10 and the previously described chocolate milk intervention ${ }^{(10)}$, the BS30 condition in the present study was protein matched to a similar bedtime snack study that found that $30 \mathrm{~g}$ of whey or casein increased next morning $\mathrm{RMR}^{(7)}$. Ormsbee et al. ${ }^{(10)}$ reported a higher RMR with the bedtime ingestion of $355 \mathrm{ml}$ of skimmed chocolate milk ( $12 \mathrm{~g}$ protein, $30 \mathrm{~g}$ carbohydrate, $0 \mathrm{~g}$ fat, $753 \mathrm{~kJ}$ ) compared with a non-energetic placebo in young, trained, lean females. By contrast, in the present study of mildly overweight males, next morning RMR was similar between milk and non-energetic control conditions, irrespective of the dose of protein and energy content in the bedtime milk snack. Multiple factors may explain these discrepant findings, including differences in time elapsed between bedtime snack ingestion and metabolic measurements and differences in participant characteristics between studies. Sleep quality can be excluded because bedtime milk ingestion had no impact on sleep duration and quality in the present study.

One plausible explanation for the inconsistent findings regarding RMR between bedtime snack ingestion studies concerns time elapsed between bedtime snack ingestion and metabolic measurements the next morning. Utilising a respiratory chamber, previous studies have demonstrated that when an evening meal was consumed at 17.30 hours and then an evening snack at 19.30 hours, the increase in energy expenditure due to diet-induced thermogenesis returned to basal levels approximately $6 \mathrm{~h}$ after ingestion of the evening snack ${ }^{(25,26)}$. Conversely, data also exist demonstrating that diet-induced thermogenesis persists for longer than $6 \mathrm{~h}^{(27)}$. In the present study, we standardised the time between consumption of a bedtime milk snack (22.30 hours) and next morning measurements of indirect calorimetry (08.10 hours) at $9 \mathrm{~h}$ and $40 \mathrm{~min}$ and observed no increase in RMR with milk ingestion. Similarly, in a study of obese men, Kinsey et al. ${ }^{(8)}$ reported no increase in next morning RMR measured about $8 \mathrm{~h}$ after bedtime ingestion of $30 \mathrm{~g}$ of casein protein compared with a non-energetic placebo. In contrast, Ormsbee et al. ${ }^{(10)}$ demonstrated next morning RMR to be increased by about $5 \%$ compared with a non-energetic placebo in lean, trained females when bedtime chocolate milk was consumed as little as $7 \mathrm{~h}$ before the measurement of RMR the following morning. As such, in the present study, we potentially missed the impact of diet-induced thermogenesis of bedtime milk ingestion on next morning RMR because we collected metabolic measurements $3 \mathrm{~h}$ and $40 \mathrm{~min}$ beyond the proposed approximately $6 \mathrm{~h}$ cut off point ${ }^{(25,26)}$. Taken together, these data suggest the time elapsed between bedtime snack ingestion and the next morning measurement of energy expenditure impacts, at least in part, the ability to detect an increase in next morning RMR through diet-induced thermogenesis.

In theory, the discrepant findings between past ${ }^{(6-9)}$ and present investigations of bedtime snack ingestion and next morning metabolism also may relate to the characteristics of recruited participants. Diet-induced thermogenesis has been reported to be greater in lean $v$. obese males ${ }^{(28)}$, which implies that bedtime snack ingestion confers a greater potential to increase next morning RMR in lean compared with obese males. Accordingly, a previous study in physically active men demonstrated an increase in RMR the following morning after bedtime ingestion of whey protein, casein protein, and carbohydrate $^{(7)}$. In contrast, a study in obese men with a BMI of $36 \cdot 1 \mathrm{~kg} / \mathrm{m}^{2}$ observed no difference in next morning RMR following bedtime ingestion of casein protein compared with a non-energetic placebo ${ }^{(8)}$. Consistent with this finding, we observed no increase in RMR the following morning after bedtime skimmed milk ingestion in overweight men with a BMI of $27.4 \mathrm{~kg} / \mathrm{m}^{2}$. Interestingly, although a previous study reported no difference in diet-induced thermogenesis between lean and obese females fed during the day ${ }^{(29)}$, other studies have reported a higher next morning RMR after bedtime snack ingestion in lean, trained females ${ }^{(10)}$, but not in obese females ${ }^{(6,9)}$ when compared with no bedtime snack ingestion at baseline. Hence, future studies should compare sex-differences in next morning RMR following bedtime snack ingestion between lean and obese individuals.

The timing of next morning metabolic measurements and blood sampling also may explain why we failed to observe any modulation of substrate utilisation with bedtime milk ingestion. Milk consists of all macronutrients, of which protein composition constitutes $80 \%$ casein and $20 \%$ whey. The bedtime ingestion of casein protein has been shown to increase fat oxidation rates the next morning compared with whey protein and carbohydrate ${ }^{(7)}$. It was speculated that the lower insulin response to ingested casein compared with whey protein and carbohydrate resulted in reduced inhibition of fat oxidation the following morning ${ }^{(7)}$. Therefore, we anticipated that bedtime milk ingestion, which is rich in casein protein, would elicit an increase in fat oxidation the following morning. However, in the present study, morning fasting glucose and insulin concentrations in both milk conditions were similar to the non-energetic placebo condition, suggesting that, as perhaps could be expected, the glucose and insulin concentrations had returned to basal levels the next morning following bedtime milk ingestion. Accordingly, we observed no differences in substrate utilisation the following morning as estimated by RER between milk and placebo conditions. We also acknowledge that, in the present study, carbohydrate and fat oxidation rates may have been overestimated given that our calculations of substrate utilisation assumed negligible protein oxidation. Previous bedtime snack studies have made the same assumption with the bedtime provision of $30 \mathrm{~g}$ of protein ${ }^{(6-9)}$. Future studies are warranted that collect overnight gas exchange measurement using a respiratory chamber to determine the time course of change in overnight energy expenditure and substrate utilisation following bedtime snack ingestion.

Given that bedtime chocolate milk ingestion elicited a reduction in appetite the following morning compared with a non-energetic placebo in lean, trained females ${ }^{(10)}$, we anticipated that bedtime skimmed milk ingestion also would promote the suppression of appetite the following morning in mildly overweight males. However, in the present study, whereas evening hunger was suppressed and fullness increased 
immediately after bedtime consumption of milk compared with a non-energetic placebo, this effect was not maintained the following morning, even in the BS30 condition. Interestingly, other bedtime snack studies examining whey, casein, and carbohydrate ingestion reported inconsistent results relating to next morning appetite ${ }^{(6-10)}$. For example, the bedtime ingestion of $30 \mathrm{~g}$ of casein has been reported to be more satiating the next morning compared with whey or carbohydrate ingestion, but conversely, was found to increase the desire to eat the next morning compared with a non-energetic placebo at bedtime ${ }^{(8)}$. Future bedtime snack studies are required to clarify the differences in next morning appetite after intake of various mixed macronutrient food sources, for example milk, compared with single macronutrient snacks, both administered in solid and liquid form. Such studies should include measurements of candidate appetite regulating hormones (e.g. ghrelin) to provide mechanistic insight into the potential role of a bedtime snack in modulating next morning appetite.

The practical implication of modulating next morning RMR, substrate utilisation and appetite with bedtime snack ingestion relates to weight management. In theory, increasing next morning RMR and decreasing appetite may contribute to an overall negative energy balance. However, the hypothesis that there is a negative correlation between RMR (or energy expenditure) and body weight remains controversial. Two longitudinal studies in Native Americans ${ }^{(30,31)}$ and another in Caucasian adults ${ }^{(32)}$ have demonstrated that lower values of energy expenditure and RMR were significant predictors of increases in body weight. Conversely, other studies observed no correlation between RMR and changes in body weight ${ }^{(33-35)}$. Therefore, in theory, increasing next morning RMR may contribute to an overall negative energy balance, but it is yet to be proven whether an association exists between RMR and changes in body weight.

In addition to obtaining subjective measurements of appetite, we also assessed subsequent energy intake the following morning using an ad libitum breakfast of cornflakes, as well as energy intake during the following $24 \mathrm{~h}$. Given that subjective hunger and desire to eat were similar between conditions, and that there was only a trend $(P=0.07)$ for an effect of bedtime snack on fullness the following morning, it follows that bedtime milk ingestion failed to modulate energy intake during the ad libitum breakfast. Interestingly, although not statistically significant $(P=0 \cdot 21)$, energy intake at breakfast for the BS30 condition was 18 and $25 \%$ higher than BSO and BS10 conditions, respectively, whereas no differences in energy intake over the $24 \mathrm{~h}$ post-trial period were observed between conditions. The bedtime milk snack interventions induced a similar pattern on next morning RMR (BS30>BS0>BS10). Taken together, these acute data are consistent with studies demonstrating that RMR is associated with ad libitum energy intake $^{(36,37)}$. It is possible that the lack of compensation in daytime energy intake when mildly overweight males consumed the most energy dense milk snack (BS30) at bedtime was related to the increase in energy intake as a result of the increased energy demands arising from the elevated post-prandial RMR. Future chronic bedtime snack intervention studies are warranted to confirm this seemingly adverse observation from a weight management perspective. Although not favourable in that respect, it is plausible that those with sarcopenia and aiming to retain lean mass, for example older adults $^{(38)}$, may benefit from chronic ingestion of a bedtime snack containing $30 \mathrm{~g}$ protein and the resulting increase in energy intake the following morning. We acknowledge that participant preference for the sole breakfast option of cornflakes may have affected their overall energy intake as no alternative food choice to cornflakes was offered at breakfast. In addition, we cannot discount the possibility that participants may have under-reported or made changes to their usual food intake $^{(39)}$ as food records were the only method employed to assess $24 \mathrm{~h}$ post-trial energy intake. Moreover, in the present study, we did not assess any eating behaviour traits such as cognitive restraint disinhibition and susceptibility to hunger in our cohort of mildly overweight young men that may have influenced the energy intake between participants during the breakfast test meal. Hence, we acknowledge that highly restrained participants may have eaten less during breakfast than participants with low restraint. Nevertheless, based on our findings, it appears that bedtime milk ingestion does not impact energy intake the following day in mildly overweight men.

Although the bedtime milk snack did not impact appetite and subsequent energy at breakfast the following morning, perhaps unsurprisingly, appetite was reduced during the evening period immediately following milk ingestion compared with placebo. Hence, it may be argued that bedtime milk ingestion could play a role in reducing energy intake before bedtime. Evidence exists to suggest that individuals with weight management issues may benefit most from controlling appetite over the evening period ${ }^{(1)}$. Night eating, defined as waking at night at least once a week to consume food and/or consuming $25 \%$ or more of total daily energy intake after the last meal of the day, has been demonstrated to be 2.5 times more prevalent in obese compared with normal weight individuals ${ }^{(2)}$. Furthermore, total daily energy intake appears to increase as energy intake increases at night between 18.00 and 02.00 hours $^{(1,40)}$. In the present study, whilst milk ingestion suppressed appetite before bedtime, no differences in appetite were observed between BS10 and BS30 conditions. Therefore, ingesting a low energy and nutrient-rich snack such as a typical $200 \mathrm{ml}$ glass of milk containing 7-10 g of protein (as in the BS10 condition in the present study) about 30 min before bedtime appears adequate to modulate appetite in the evening and may serve to displace intake of potentially energy dense foods that can contribute to higher total daily energy intake. This notion is supported by a study in which overweight or obese participants with self-reported night snacking behaviours were instructed to consume a fixed readyto-eat cereal with milk daily $90 \mathrm{~min}$ after the evening meal ${ }^{(41)}$. After 4 weeks of the intervention, participants who complied with the daily evening snack protocol significantly reduced their post-evening meal energy intake, resulting in a trend towards greater body weight reduction compared with participants who continued on their normal diet ${ }^{(41)}$. Participants in the present study consumed each bedtime snack treatment on one occasion only. Hence future studies are warranted to investigate if the chronic ingestion of low energy and nutrient-dense bedtime snack can contribute to weight management, without long-term implications on cardiometabolic health. 
To conclude, the bedtime ingestion of milk containing 10 or $30 \mathrm{~g}$ of protein does not make an impact on RMR, substrate utilisation and appetite the following morning ( $>9 \mathrm{~h}$ postprandial) compared with a non-energetic placebo snack in mildly overweight males. Consequently, energy intake in the subsequent breakfast and $24 \mathrm{~h}$ post-trial period was similar between conditions. To date, findings from bedtime snack studies have been inconsistent, rendering the role of bedtime energy intake as a potential weight management strategy inconclusive. Future studies that include chronic bedtime energy intake of foods with different macronutrient composition and texture are warranted to characterise the long-term implications of a structured bedtime snack $v$. free living bedtime eating habits. The best time of day to consume a milk-based snack to suppress appetite and reduce subsequent energy intake also remains unknown.

\section{Acknowledgements}

The authors thank the study participants for their dedication in the present study. They also thank Thomas Di Virgilio, Jordan Philpott, Nidia Rodriguez, Connor Davidson, Kate McDougall and Amanda Nicol for their help with data collection.

No funding was received for the present study.

A. H. H. L., D. R. C., T. G. C., S. D. R. G., K. D. T. and O. C. W. conceptualised and designed the research. A. H. H. L., G. M. D. and O. C. W. conducted the research, while A. H. H. L. and G. M. D. analysed the data. A. H. H. L. and O. C. W. wrote the paper and had primary responsibility for the final content. All authors read, edited and approved the final manuscript.

The authors have no conflicts of interest to declare.

\section{References}

1. de Castro JM (2004) The time of day of food intake influences overall intake in humans. J Nutr 134, 104-111.

2. Tholin S, Lindroos A, Tynelius P, et al. (2009) Prevalence of night eating in obese and nonobese twins. Obesity (Silver Spring) 17, 1050-1055.

3. Lennernäs M, Akerstedt T \& Hambraeus L (1994) Nocturnal eating and serum cholesterol of three-shift workers. Scand J Work Environ Health 20, 401-406.

4. Romon M, Edme JL, Boulenguez C, et al. (1993) Circadian variation of diet-induced thermogenesis. Am J Clin Nutr 57, 476-480.

5. Kinsey AW \& Ormsbee MJ (2015) The health impact of nighttime eating: old and new perspectives. Nutrients 7, 2648-2662.

6. Kinsey AW, Eddy WR, Madzima TA, et al. (2014) Influence of night-time protein and carbohydrate intake on appetite and cardiometabolic risk in sedentary overweight and obese women. Br J Nutr 112, 320-327.

7. Madzima TA, Panton LB, Fretti SK, et al. (2014) Night-time consumption of protein or carbohydrate results in increased morning resting energy expenditure in active collegeaged men. Br J Nutr 111, 71-77.

8. Kinsey AW, Cappadona SR, Panton LB, et al. (2016) The effect of casein protein prior to sleep on fat metabolism in obese men. Nutrients $\mathbf{8}, 452$.

9. Ormsbee MJ, Kinsey AW, Eddy WR, et al. (2015) The influence of nighttime feeding of carbohydrate or protein combined with exercise training on appetite and cardiometabolic risk in young obese women. Appl Physiol Nutr Metab 40, 37-45.

10. Ormsbee MJ, Gorman KA, Miller EA, et al. (2016) Nighttime feeding likely alters morning metabolism but not exercise performance in female athletes. Appl Physiol Nutr Metab $\mathbf{4 1}$, 719-727.

11. Pennings B, Boirie Y, Senden JMG, et al. (2011) Whey protein stimulates postprandial muscle protein accretion more effectively than do casein and casein hydrolysate in older men. Am J Clin Nutr 93, 997-1005.

12. Trommelen J \& van Loon LJC (2016) Pre-sleep protein ingestion to improve the skeletal muscle adaptive response to exercise training. Nutrients $\mathbf{8}, 763$.

13. Pereira PC (2014) Milk nutritional composition and its role in human health. Nutrition 30, 619-627.

14. Sports Dietitians Australia (2011) Fact sheet protein and amino acid supplementation. https://www.sportsdietitians.com.au/ wp-content/uploads/2015/04/110701-Protein-Supplementation_ General.pdf (accessed June 2017).

15. Dougkas A, Minihane AM, Givens DI, et al. (2012) Differential effects of dairy snacks on appetite, but not overall energy intake. Br J Nutr 108, 2274-2285.

16. Dove ER, Hodgson JM, Puddey IB, et al. (2009) Skim milk compared with a fruit drink acutely reduces appetite and energy intake in overweight men and women. Am J Clin Nutr 90, 70-75.

17. Halton TL \& Hu FB (2004) The effects of high protein diets on thermogenesis, satiety and weight loss: a critical review. J Am Coll Nutr 23, 373-385.

18. Phillips SM, Chevalier S \& Leidy HJ (2016) Protein 'requirements' beyond the RDA: implications for optimizing health. Appl Physiol Nutr Metab 41, 565-572.

19. GOV.UK. (2014) NDNS: results from Years 1 to 4 (combined). https://www.gov.uk/government/statistics/national-diet-andnutrition-survey-results-from-years-1-to-4-combined-of-therolling-programme-for-2008-and-2009-to-2011-and-2012 (accessed February 2017).

20. Weir JBDB (1949) New methods for calculating metabolic rate with special reference to protein metabolism. J Physiol 109, 1-9.

21. Flint A, Raben A, Blundell JE, et al. (2000) Reproducibility, power and validity of visual analogue scales in assessment of appetite sensations in single test meal studies. Int J Obes Relat Metab Disord. 24, 38-48.

22. Spaeth AM, Dinges DF \& Goel N (2015) Resting metabolic rate varies by race and by sleep duration. Obesity (Silver Spring) 23, 2349-2356.

23. Parrott AC \& Hindmarch I (1978) Factor analysis of a sleep evaluation questionnaire. Psychol Med 8, 325-329.

24. Diabetes Trials Unit - University of Oxford (2013) HOMA Calculator: overview. https://www.dtu.ox.ac.uk/homacalcu lator/ (accessed July 2017).

25. Westerterp KR (2004) Diet induced thermogenesis. Nutr Metab 1, 5 .

26. Verboeket-van de Venne WP, Westerterp KR, HermansLimpens TJ, et al. (1996) Long-term effects of consumption of full-fat or reduced-fat products in healthy non-obese volunteers: assessment of energy expenditure and substrate oxidation. Metabolism 45, 1004-1010.

27. Reed GW \& Hill JO (1996) Measuring the thermic effect of food. Am J Clin Nutr 63, 164-169.

28. Segal KR, Edaño A \& Tomas MB (1990) Thermic effect of a meal over 3 and 6 hours in lean and obese men. Metabolism 39, 985-992.

29. Tentolouris N, Pavlatos S, Kokkinos A, et al. (2008) Diet-induced thermogenesis and substrate oxidation are not different between lean and obese women after two different isocaloric meals, one rich in protein and one rich in fat. Metabolism 57, 313-320. 
30. Ravussin E, Lillioja S, Knowler WC, et al. (1988) Reduced rate of energy expenditure as a risk factor for body-weight gain. $N$ Engl J Med 318, 467-472.

31. Piaggi P, Thearle MS, Bogardus C, et al. (2013) Lower energy expenditure predicts long-term increases in weight and fat mass. J Clin Endocrinol Metab 98, E703-E707.

32. Buscemi S, Verga S, Caimi G, et al. (2005) Low relative resting metabolic rate and body weight gain in adult Caucasian Italians. Int J Obes 29, 287-291.

33. Seidell JC, Muller DC, Sorkin JD, et al. (1992) Fasting respiratory exchange ratio and resting metabolic rate as predictors of weight gain: the Baltimore Longitudinal Study on Aging. Int $J$ Obes Relat Metab Disord 16, 667-674.

34. Weinsier RL, Nelson KM, Hensrud DD, et al. (1995) Metabolic predictors of obesity. Contribution of resting energy expenditure, thermic effect of food, and fuel utilization to four-year weight gain of post-obese and never-obese women. J Clin Invest 95, 980-985.

35. Katzmarzyk PT, Pérusse L, Tremblay A, et al. (2000) No association between resting metabolic rate or respiratory exchange ratio and subsequent changes in body mass and fatness: 5-1/2 year follow-up of the Québec family study. Eur J Clin Nutr 54, 610-614.

36. Caudwell P, Finlayson G, Gibbons C, et al. (2013) Resting metabolic rate is associated with hunger, self-determined meal size, and daily energy intake and may represent a marker for appetite. Am J Clin Nutr 97, 7-14.

37. Piaggi P, Thearle MS, Krakoff J, et al. (2015) Higher daily energy expenditure and respiratory quotient, rather than fatfree mass, independently determine greater ad libitum overeating. J Clin Endocrinol Metab 100, 3011-3020.

38. Keller K \& Engelhardt M (2014) Strength and muscle mass loss with aging process. Age and strength loss. Muscles Ligaments Tendons J 3, 346-350.

39. Ortega RM, Pérez-Rodrigo C \& López-Sobaler AM (2015) Dietary assessment methods: dietary records. Nutr Hosp $\mathbf{3 1}$, Suppl. 3, 38-45.

40. de Castro JM (2007) The time of day and the proportions of macronutrients eaten are related to total daily food intake. Br J Nutr 98, 1077-1083.

41. Waller SM, Vander Wal JS, Klurfeld DM, et al. (2004) Evening ready-to-eat cereal consumption contributes to weight management. J Am Coll Nutr 23, 316-321. 